\title{
"Meta-atomless" architecture based on an irregular continuous fabric of coupling-tuned identical nanopillars enables highly efficient and achromatic metasurfaces
}

Cite as: Appl. Phys. Lett. 118, 081105 (2021); https://doi.org/10.1063/5.0040365

Submitted: 12 December 2020 - Accepted: 03 February 2021 • Published Online: 25 February 2021

(iD) H. Bilge Yağcı and (D) Hilmi Volkan Demir

\section{COLLECTIONS}

Paper published as part of the special topic on Metastructures: From Physics to Application
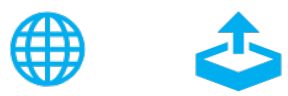

\section{ARTICLES YOU MAY BE INTERESTED IN}

Will flat optics appear in everyday life anytime soon?

Applied Physics Letters 118, 100503 (2021); https://doi.org/10.1063/5.0039885

Wavefront shaping and modulation with resonant electro-optic phase gradient metasurfaces Applied Physics Letters 118, 071104 (2021); https://doi.org/10.1063/5.0039873

Spectral imaging of flexible terahertz coding metasurface

Applied Physics Letters 118, 081101 (2021); https://doi.org/10.1063/5.0043481

田QBLOX

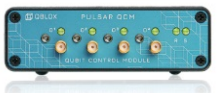

1 qubit
Shorten Setup Time Auto-Calibration More Qubits

Fully-integrated Quantum Control Stacks Ultrastable DC to $18.5 \mathrm{GHz}$ Synchronized $<<1 \mathrm{~ns}$ Ultralow noise

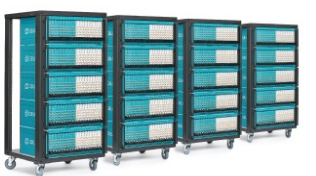

100s qubits

visit our website > 


\title{
"Meta-atomless" architecture based on an irregular continuous fabric of coupling-tuned identical nanopillars enables highly efficient and achromatic metasurfaces
}

Cite as: Appl. Phys. Lett. 118, 081105 (2021); doi: 10.1063/5.0040365

Submitted: 12 December 2020 - Accepted: 3 February 2021 .

Published Online: 25 February 2021

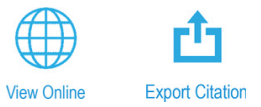

H. Bilge Yağcı ${ }^{1}$ (D) and Hilmi Volkan Demir ${ }^{1,2,3, a)}$ (iD

\begin{abstract}
AFFILIATIONS
${ }^{7}$ Department of Electrical and Electronics and UNAM-Institute of Materials Science and Nanotechnology, Bilkent University, TR-06800 Ankara, Turkey

${ }^{2}$ Department of Physics, Bilkent University, TR-06800 Ankara, Turkey

${ }^{3}$ LUMINOUS! Centre of Excellence for Semiconductor Lighting and Displays, School of Electrical and Electronic Engineering, School of Physical and Mathematical Sciences, School of Materials Science and Engineering, Nanyang Technological University, 50 Nanyang Avenue, 639798 Singapore, Singapore
\end{abstract}

Note: This Paper is part of the APL Special Collection on Metastructures: From Physics to Applications.

a) Author to whom correspondence should be addressed: volkan@stanfordalumni.org

\begin{abstract}
Metasurfaces are subwavelength-thick constructs, consisting of discrete meta-atoms, providing discretized levels of phase accumulation that collectively approximate a designed optical functionality. The meta-atoms utilizing the Pancharatnam-Berry phase with polarizationconverting structures produced encouraging implementations of optical components including metalenses. However, to date, a pending and fundamental problem of this approach has been the low device efficiency that such resulting metasurface components suffer, an unwanted side effect of large lattice constants that are used for preventing intercoupling of their meta-atoms. Although the use of near-field coupling for tuning electromagnetic resonances found its use in constructing efficient narrow-band designs, such structures fell short of providing high efficiency over a broad spectrum. Here, we propose and show that tightly packed fabrics of identical dielectric nanopillar waveguides with continuously tuned intercoupling distances make excellent and complete achromatic metasurface elements. This architecture enables the scatterers to interact with the incoming wave extremely efficiently. As a proof-of-concept demonstration, we showed an achromatic cylindrical metalens, constructed from strongly coupled dielectric nanopillars of a single geometry as continuously set phase elements in a metaatomless fashion, working in the entirety of the $400-700 \mathrm{~nm}$ band. This metalens achieves over $85 \%$ focusing efficiency across this whole spectral range. To combat polarization sensitivity, we used hexagonally stacked nanopillars to build up a polarization-independent scatterer library. Finally, a circular metalens with polarization-independent operation and achromatic focusing was obtained. This is a paradigm shift in making an achromatic metasurface architecture by weaving identical nanopillars coupled into an irregular lattice laterally constructed via carefully tuned near-field coupling.
\end{abstract}

Published under license by AIP Publishing. https://doi.org/10.1063/5.0040365

Metasurfaces are subwavelength-thick layers of engineered structures, formed to shape wavefronts. Originally, the concept has its roots in phased array antennas, in which the signal fed to an element of the array has location-dependent phase shift with respect to the reference signal. Early examples of metasurfaces started with LC oscillators ${ }^{1}$ and as operation frequencies grew larger, plasmonic ${ }^{2-5}$ and all-dielectric designs $^{6-8}$ came into being. Holograms, ${ }^{8-10}$ orbital angular momentum (OAM) manipulating devices, ${ }^{11-13}$ lenses, ${ }^{7,14-16}$ and various other functional devices ${ }^{17-19}$ have been implemented with metasurfaces. Constituting elements, dubbed as metacells (or meta-atoms), function as signal processing units in space via their positiondependent phase response and vary in geometry and material with design spectra and functionality. The Pancharatnam-Berry (PB) phase $^{20}$ and/or resonances in the metacells can be utilized to obtain 
the phase coverage required. Resonances in metacells were used in the very first metasurface designs as high-Q scattering mechanisms. ${ }^{21}$ Due to the nature of the resonance-tuning approach, these structures were able to offer high functional efficiency only for limited spectral band operation and, thus, narrow-band applications. ${ }^{22,23}$ As the attention of the photonics community shifted toward achromatic focusing, resonances became insufficient as the dominant phase contributors. Although subsequent attempts to widen operating bandwidth with multiwavelength designs ${ }^{24-26}$ did not suffice to justify using only resonance tuning, the reported use case of weak resonances for compensation of material dispersion ${ }^{27}$ was quickly adapted into meta-atom designs and employed extensively. The PB phase, on the other hand, provided a frequency-independent phase accumulation mechanism, accompanied by an undesired polarization dependency. While it was possible to exploit polarization dependency in certain applications, ${ }^{28,29}$ achromatic operation of metalenses with the PB phase was shadowed by low polarization conversion efficiency, ${ }^{30}$ which was shown to be limited by a theoretical maximum. ${ }^{2}$ Efficient achromatic focusing with metalenses has remained a challenge as a result to date.

Due to the impressive power and flexibility of the metasurface concept as an optical signal processing tool, there exists a primordial design constraint to satisfy uncoupled operation. Uncoupled operation eases the design process but suffers two major flaws: first, the required phase function is sampled on the design to select the most suitable elements at each unit cell's center. Commonly, the lattice constant $\Lambda$ is chosen as the inverse of Nyquist rate to faithfully construct the desired wavefront, a bare minimum when the meta-atom response perfectly matches the required functionality. Second, in analysis and optimization of the utilized scattering mechanism, intercell coupling is often modeled as a parasitic effect, its suppression requiring restrictions in

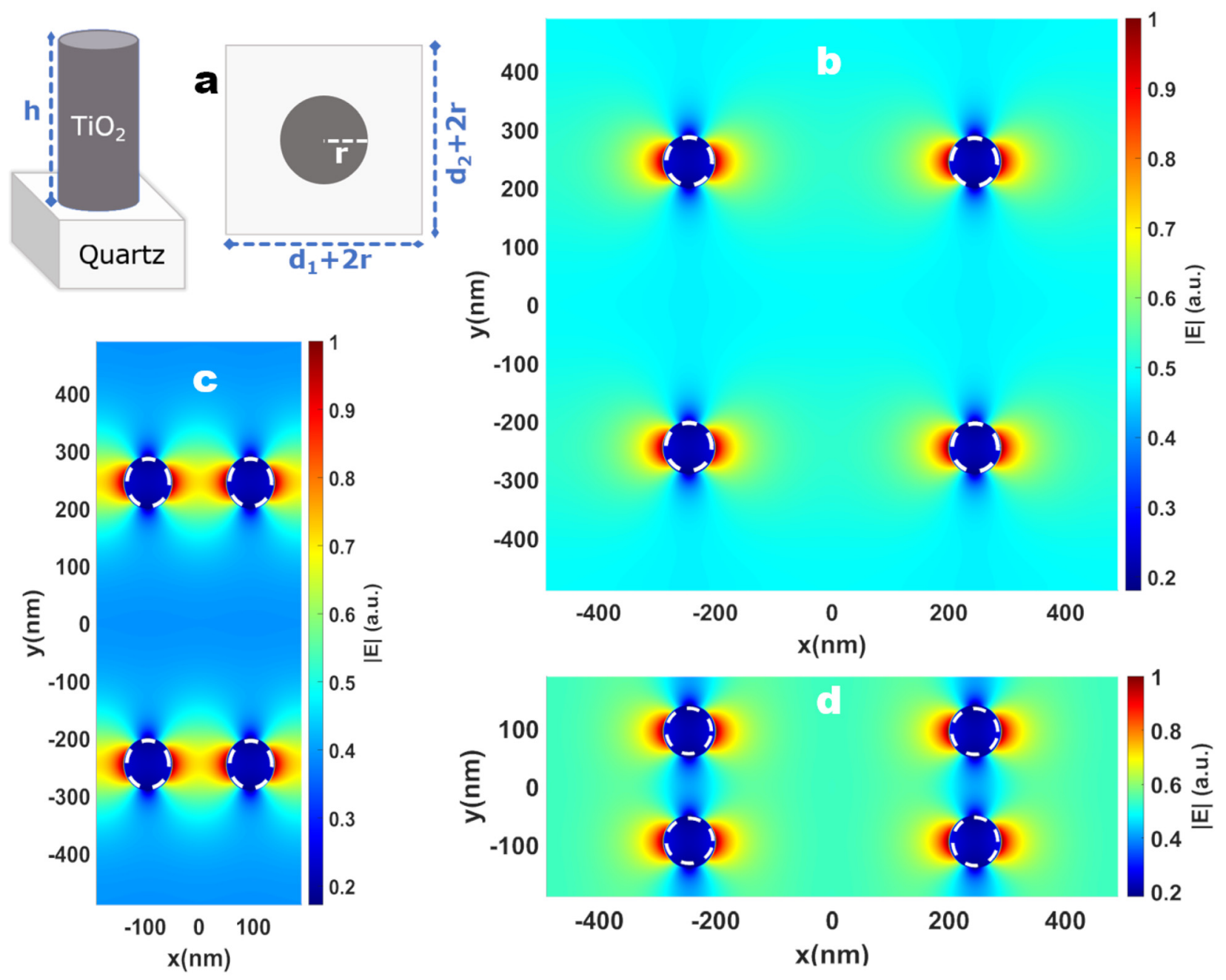

FIG. 1. (a) An exemplary scatterer defined with geometrical parameters $r$ and $h$ and lattice parameters $d_{1}$ and $d_{2}$. (b)-(d) Horizontal slice of electric field components along the input polarization for nanopillars $(\{r, h\}=\{45 \mathrm{~nm}, 600 \mathrm{~nm}\})$ in specified lattices. Nanopillars are superimposed on the fields with white lines. To visualize field coupling better, the simulated periodic cell region is constructed from four closely located nanopillars. (b) Uncoupled case $\left(\left\{d_{1}, d_{2}\right\}=\{400 \mathrm{~nm}, 400 \mathrm{~nm}\}\right)$. (c) Coupled along the input polarization (parallel-coupled) $\left(\left\{d_{1}, d_{2}\right\}=\{100 \mathrm{~nm}, 400 \mathrm{~nm}\}\right)$. (d) Coupled orthogonal to the input polarization (orthogonal-coupled) $\left(\left\{d_{1}, d_{2}\right\}=\{400 \mathrm{~nm}, 100 \mathrm{~nm}\}\right)$. 
the design space. While the idea of uncoupled operation is important for the design of spatially independent scatterers, achromatic lensing does not inherently benefit from it: the amplitude response should ideally be unity for all scatterers and the phase function required for achromatic focusing should be monotonic in space and frequency; therefore, it should be possible to design an efficient metalens with such constraints relaxed.

To retain achromatic operation in a wide spectral range, the dispersion between the responses of the metasurface library elements should be kept constant. In a previous study conducted in our group, dielectric pillars were shown to be suitable achromatic phase elements when operated outside of the particle resonances. ${ }^{31}$ Unfortunately, the effectiveness of this approach diminishes in the visible range due to complications in the waveguide dispersion. Here, we hypothesize that strong near-field coupling between scatterers results in substantially enhanced interactivity with the incoming wave and achieves superior efficiency, while providing dispersion compensation via eliminating group dispersion delay and ensuring achromatic operation. We propose closely coupled dielectric nanopillars with continuously tuned interactivity as a paradigm shift in building a scatterer architecture of metasurfaces, which we coin as meta-atomless metasurfaces (MAMs) here. We intentionally avoid any internal or external resonances to guarantee achromaticity and rely only on the coupling between our nanopillars operating in guiding mode. As a proof-of-concept, we

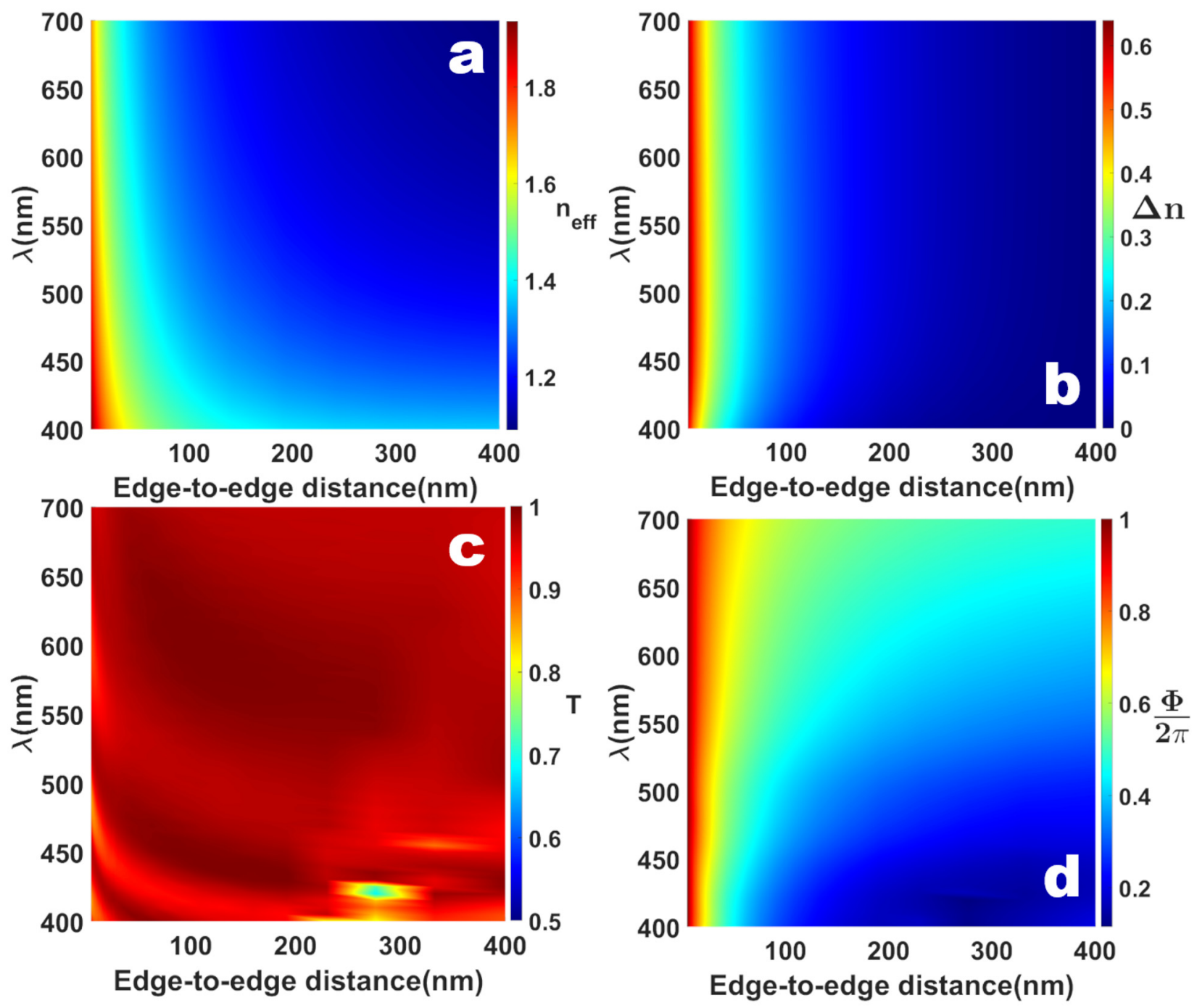

FIG. 2. Numerical simulation results for a lattice of $\mathrm{TiO}_{2}$ nanopillars. Stacking is along the input polarization. The edge-to-edge distance in the orthogonal direction is $30 \mathrm{~nm}$. (a) and (b) Simulation results for an array of infinite cylinders suspended in vacuum, done in MODE Waveguide Simulator. (a) $n_{\text {eff }}$ vs $\lambda$ and $d_{1}$ for the guided mode, polarized along the input polarization. (b) Effective index difference map. For all $\lambda$, the parallel-uncoupled case is taken as a reference. The index difference is constant for changing wavelengths for most of the spectrum, implying achromaticity. (c) and (d) FDTD results for $600 \mathrm{~nm}$-high $\mathrm{TiO}_{2}$ nanopillars, residing on a quartz substrate, with the same lattice setup in (a) and (b). (c) Transmission map of the nanopillars. (d) Phase response of the nanopillars. 
show an efficient achromatic cylindrical MAM lens composed of identical dielectric nanopillars utilizing the edge-to-edge distance as the phase elements. This conceptual architecture enables $86 \%$ focusing efficiency for the $400-700 \mathrm{~nm}$ range, a record value across such a broadband spectrum. As the next step, we construct another library of nanopillars with honeycomb stacking, which provides us with the ability to build up a circular MAM lens working in the same spectral region with completely polarization-insensitive operation. Our highly efficient structures prove the legitimacy of coupling-tuning as a means to construct achromatic phase-accumulating elements.

First, we systematically studied the effect of coupling to the field profiles of the guided modes of the nanopillars. With the decreasing interpillar distance, weakly guided modes of the uncoupled nanopillars start to interact with each other, increasing the field amplitudes in the surrounding medium. Such an increase in field amplitudes can be thought as an additional index, justified by the effective index formulation for a guided mode. ${ }^{32}$ In turn, this coupling-dependent effective index change allows us to form phase elements for MAM. Additionally, as the fields are localized in the gap, the coupled operation increases the interactivity of the scatterers with the incoming wave, effectively increasing their scattering efficiency.

Figure 1(a) shows an illustration of the scatterers, with $h$ being the height of each nanopillar and $d_{1}$ and $d_{2}$ denoting the edge-to-edge distances between the nearest nanopillars in the respective directions along and perpendicular to the input polarization. Figures 1 (b)-1(d) visualize electric field profiles along the input polarization for $\lambda=700 \mathrm{~nm}$, normalized to the field maxima for each case. In the uncoupled case [Fig. 1(b)], nanopillars hardly interact with each other. When nanopillars are coupled along the input polarization (dubbed parallel-coupled case), shown in Fig. 1(c), interactions between nanopillars are visible and fields are confined in a smaller region, a precursor of field enhancement or increase in effective index. Similarly, coupling nanopillars orthogonal to the input polarization (dubbed orthogonal-coupled case) in Fig. 1(d) creates localization, and while the mode is more confined than the uncoupled case, it is weaker than the parallel-coupled case. Such a phenomenon can be explained with breaking of mode degeneracy, observed in nanopillar dimers. ${ }^{33}$

To test our hypothesis, we next studied periodic nanopillars with fixed $r=45 \mathrm{~nm}$ and $d_{2}=30 \mathrm{~nm}$ while $d_{1}$ was used as an independent parameter. The obtained effective index map for the parameter space can be seen in Fig. 2(a). Using the parallel-uncoupled case as a reference, we obtained an index difference map that unfolds achromatic nature of the coupling [Fig. 2(b)]. Although a small dispersion mismatch exists toward the short-wavelength limit that stems from the unbalanced compensation of dispersion for $d_{2}=30 \mathrm{~nm}$, this represents the best trade-off between flatness and magnitude of index difference. These index difference results can be translated to the phase differences acquired in $\Delta h$ distance by $\Phi=2 \pi \mathrm{n}_{\text {eff }} \frac{\Delta h}{2}$. To validate the waveguiding approach, the responses of the nanopillars were also obtained via finite-difference time-domain (FDTD) computations. In
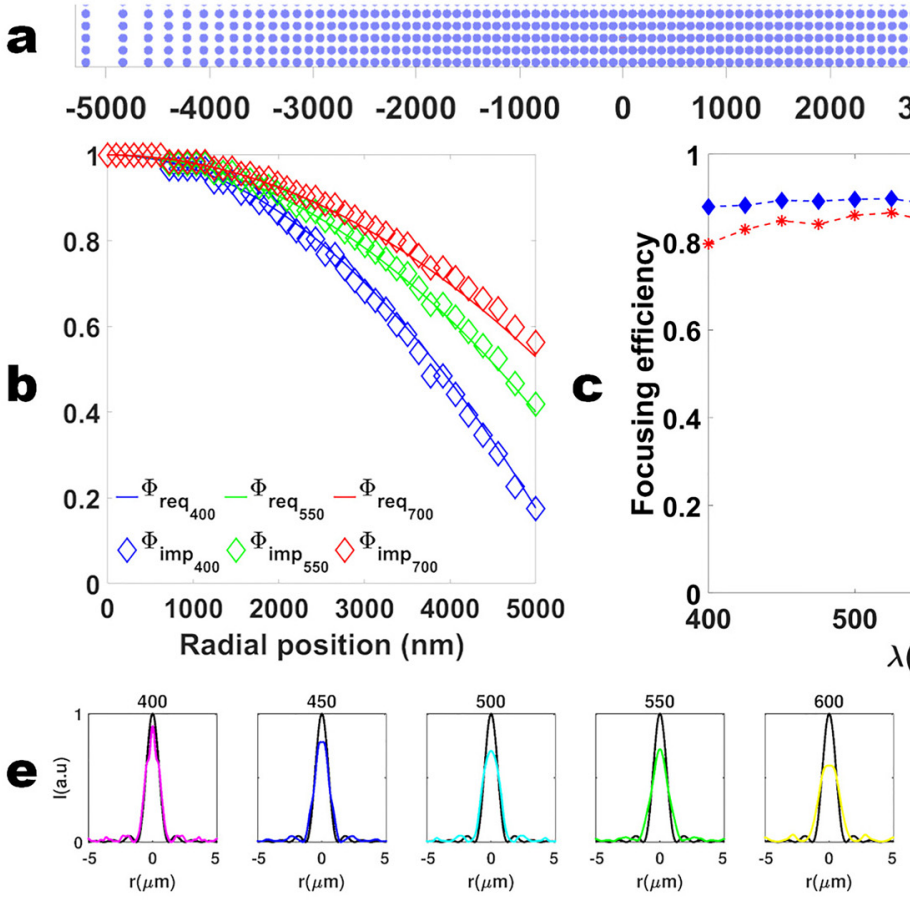

FIG. 3. Design and characterization of the cylindrical MAM lens. (a) Placement of nanopillars on the design plane. Variation in $d_{1}$ is used for constructing phase elements, while $d_{2}$ is constant with $30 \mathrm{~nm}, r=45 \mathrm{~nm}$. (b) Required phase response for the design $\Phi_{\text {req, }}$ and realized phase response $\Phi_{i m p}$ for $\lambda=\{400 \mathrm{~nm}, 550 \mathrm{~nm}, 700 \mathrm{~nm}\}$. (c) Relative and absolute focusing efficiency of the design. Both efficiencies are above $80 \%$ over the spectral band, showing the capability of the proposed structure as a highly efficient metalens. (d) False-colored image of normalized intensities along the optical axis. The white dashed line shows the design focus at $z=32 \mu \mathrm{m}$. (e) Wavelength-specific Strehl ratios, normalized to slit diffraction pattern. The dashed line marks a Strehl ratio of 0.8 . 
Fig. 2(c), optical transmission through the scatterer is shown over the wavelength with varying edge-to-edge distances. High transmission throughout the parameter space stems from the lack of cutoff for the fundamental mode in cylindrical waveguides and high coupling of the incident wave to the guided modes; small ripples in the transmission can be thought of reflection from an effective medium slab. Minimum for the transmission does not fit to this explanation and is mostly a consequence of dipole resonances. The phase difference variation over $\lambda$ with respect to $d_{1}$ [Fig. 2(d)] exhibits a great similarity to Fig. S1, confirming the validity of our approach.

To verify the applicability of rectangular-packed structures, here we implemented a cylindrical MAM lens with $f=32 \mu \mathrm{m}$ and $\mathrm{NA}=0.154$, following the formulations of the phase requirements of a cylindrical lens presented in the supplementary material. Utilizing Eq. (S4), we acquired an $r$-dependent edge-to-edge distance map, which is used for placing identical nanopillars on the lens plane [Fig. 3(a)]. At each axial position, the required phase $\Phi_{\text {req }}$ is sampled by the actual imposed phase $\Phi_{i m p}$ with consistency over the design spectra [Fig. 3(b)]. As shown in Fig. 3(c), the proposed structure focuses efficiently across the entire bandwidth, with the focusing efficiencies over $85 \%$ and the absolute efficiency levels over $80 \%$. False-colored images of intensities along the optical axis of the lens are displayed in Fig. 3(d), normalized with respect to the intensity maxima at each wavelength. While our architecture is diffraction-limited for the short-wavelength limit, its ability to resolve finer details suffers as the wavelength increases. The extended depth-of-focus for all wavelengths allows one to optimize for a more uniform resolution at all wavelengths without a significant loss in the focal spot intensity.

Tuning the coupling in only one direction, as we did in our cylindrical MAM lens, imposes a polarization dependency to the design (see the supplementary material for further discussion). To combat this, we can also stack the nanopillars in a honeycomb lattice. While a honeycomb lattice of nanopillars is not fourfold symmetric specifically, its response approximates a circularly symmetric scatterer as the length scales shrink, allowing us to design polarization-insensitive scatterers without losing performance. As shown in Figs. 4(a)-4(f), a honeycomb lattice with $r=45 \mathrm{~nm}$ has similar qualities to the rectangular-coupled nanopillars in Fig. 2: it possesses a similarly flat dispersion curve apparent from the almost $\lambda$-independent nature of the effective index difference map shown in Fig. 4(b). Effective index
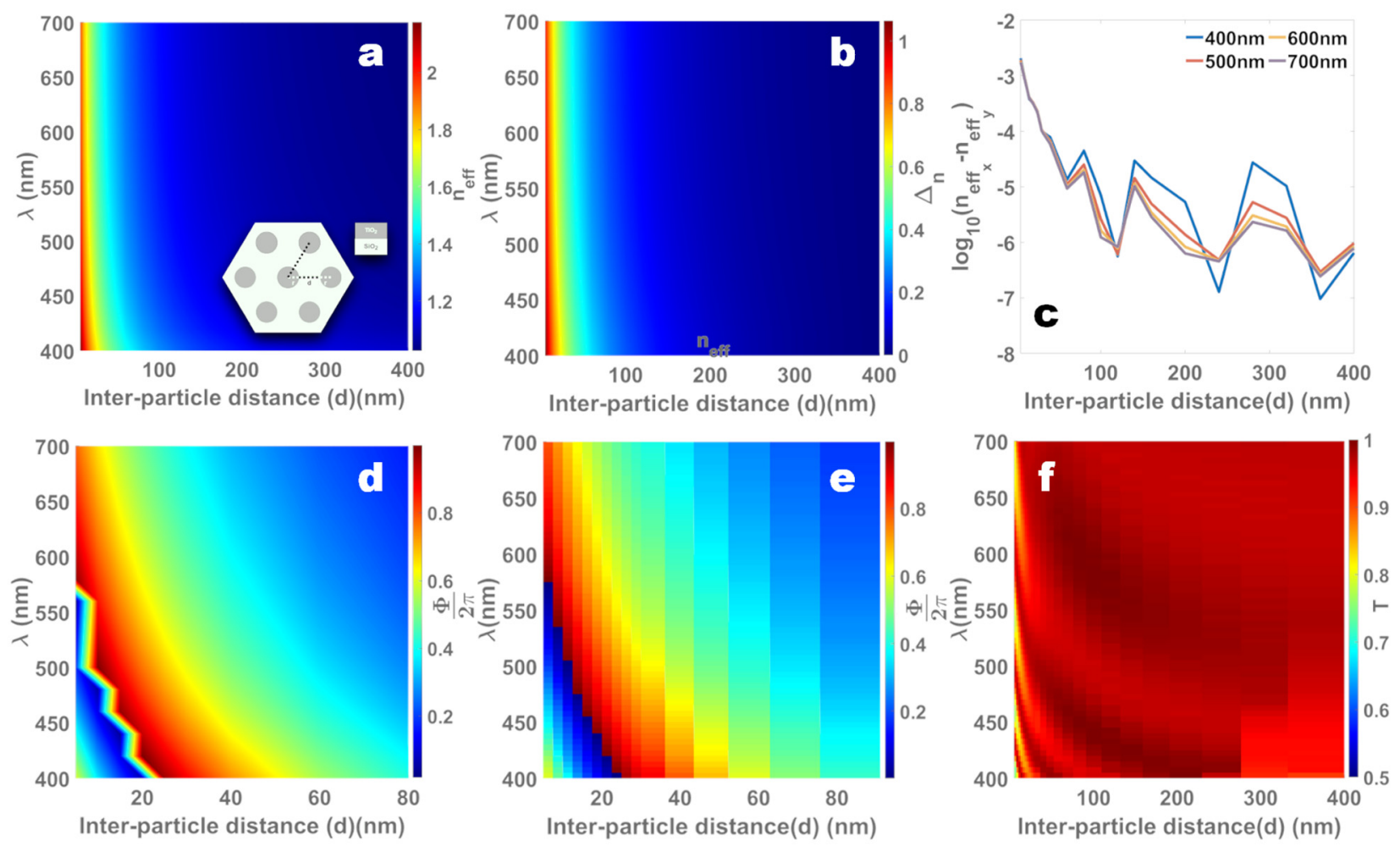

FIG. 4. Phase response and transmission pattern of nanopillars stacked in the honeycomb lattice. (a)-(d) MODE simulations for nanopillars stacked in the honeycomb lattice, with $r=45 \mathrm{~nm}$ and interparticle distance $d$ chosen as the independent parameter. (a) Effective index map for honeycomb stacking. Excitation polarization is chosen parallel to the horizontal direction. Inset: illustration of nanopillars in a honeycomb lattice. (b) Effective index difference map for the geometry used in part (a). Higher effective index difference compared to the rectangular stacking of nanopillars follows from a relatively high fill factor and coupling strength. (c) Effective index differences between the horizontally polarized and the vertically polarized excitation. For all design spectra, the index difference between these polarizations is less than $5 \%$. (d) Calculated phase response map for the lattice in (a), obtained via $\Phi=2 \pi \mathrm{n}_{e f f} \frac{\Delta h}{2}$ with $\Delta h=600 \mathrm{~nm}$, normalized to the maximally uncoupled case $(d=400 \mathrm{~nm})$. (e) and (f) FDTD simulation results for $600 \mathrm{~nm}$-high nanopillars standing on a quartz substrate. Input excitation is aligned with the horizontal direction. (e) Phase response map of the honeycomb lattice, normalized to the maximally uncoupled case. (f) Transmission map of the honeycomb lattice. 
differences between orthogonal excitations are negligible with $<1 \%$ difference for most of the spectrum as seen in Fig. 4(c). Figures 4(d) and 4(e) show a comparison between the waveguide phase [Fig. 4(d)], calculated from the effective index map, and the phase response acquired via FDTD computations [Fig. 4(e)]. The striking similarity of two approaches in terms of the shape and the magnitude of the phase response supports the validity of our approximation. The honeycomb lattice is also highly transmittive, as seen in Fig. 4(e). Subsequently, we designed a circular, achromatic, and polarization-insensitive MAM lens with $\mathrm{NA}=0.26$ and $f=11 \mu \mathrm{m}$. A relatively small lens diameter was chosen to impose a fine mesh while respecting computational limitations. To minimize the placement errors originating from the tiling of our irregular lattice, we utilized an optimization algorithm (see the supplementary material for details). The resultant architecture and its characteristics are shown in Fig. 5.

As seen in Fig. 5(a), phase requirements for this metalens constituted of such a continuously tuned lattice of intercoupled nanopillars as described above are met with little to no mismatch, with $\lambda=550 \mathrm{~nm}$ presenting the best match. Focusing characteristics under orthogonal input polarizations [Fig. 5(b)] exhibit a small contrast between X- and Y-polarized cases. Instead of presenting absolute efficiency, total transmission from the lens is portrayed to avoid congestion in the figure. The mismatch might be the result of a slight disturbance in the fourfold symmetry of the nanopillar placement on lens plane. Relatively low focusing efficiency compared to cylindrical lens is caused by relatively strong side-lobes, visible in the focal spot profiles. $\lambda$-specific beam patterns and Strehl ratios are displayed in Figs. 5(c) and 5(d) with false coloring. As seen in Fig. 5(c), the focal spot of the obtained metalens is invariant to changes in wavelength, while its beam width and depth of focus have a linear dependence. This metalens also features superior resolution, seen in Fig. 5(d). Diffraction-limited values for the Strehl ratio are achieved for all wavelengths, with relatively enhanced performance in the long-wavelength limit. This is attributed to a smaller phase mismatch at longer wavelengths.

In Table I, we compare our achromatic MAM lenses with the recent works reported in the field. ${ }^{15,16,30,34,35}$ The proposed cylindrical MAM lens outperforms all the other designs in efficiency, whereas the circular MAM lens is highly diffraction-limited and achromatic and has polarization-insensitive operation. Here, the high absolute efficiencies of our devices prove the effectiveness of our MAM paradigm in wavefront manipulation.

In summary, we have proposed and demonstrated that a continuously tuned fabric of interscatterer coupling can be utilized as an achromatic phase accumulation mechanism for geometries operating in guiding mode. This paradigm allows us to devise metasurfaces that interact with the incoming wave much more effectively than metalenses of similar functionalities previously reported in the literature, all
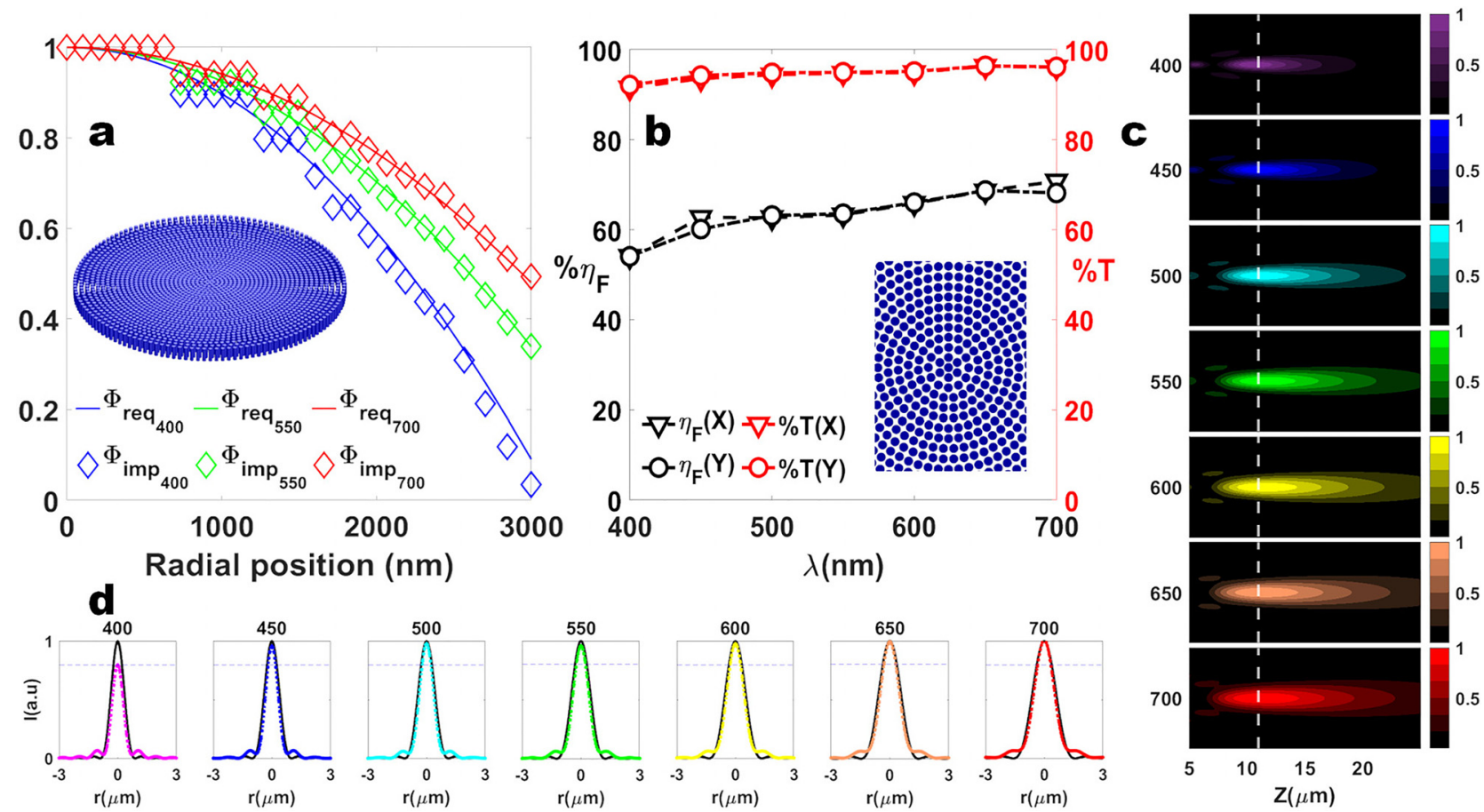

FIG. 5. Proof-of-concept demonstration of a circular, achromatic, and polarization-insensitive lens, with $N A=0.26$ and $f=11 \mu \mathrm{m}$, implemented based on MAM paradigm. (a) Phase requirements of the lens at the band edges and the mid-band, shown with $\Phi_{r e q_{\lambda}}$, plotted alongside the realized phase response at the same wavelengths, $\Phi_{i m p}$. Inset: perspective view of a circular lens. (b) Focusing efficiencies $\eta_{F}$ and total transmissions for $X$ and $Y$-polarized excitations. Total transmissions from the monitors can be translated to the absolute efficiencies by applying $\eta_{a b s_{x, y}}=\eta_{F_{x, y}} * T_{x, y}$. Inset: close-up image of the metalens in the central region. (c) False-colored image of the normalized intensities along the optical axis. The white dashed line annotates the focal plane at $f=11 \mu \mathrm{m}$. (d) Wavelength-specific Strehl ratios, calculated according to the diffraction pattern from a circular aperture. The dashed line marks the diffraction-limited value of Strehl ratio (0.8). Relatively large deviations from high Strehl ratios in the short-wavelength limit may be caused by the larger $\Phi_{\text {requ0 }}-\Phi_{\text {imp } 400}$ mismatch, shown in (a). 
TABLE I. Performance metrics of previously reported achromatic metalenses and their comparison to our MAM lenses.

\begin{tabular}{|c|c|c|c|c|c|c|}
\hline & $\frac{\text { FWHM spot size }\left(f_{\text {center }}\right)}{\text { Diffraction limit }\left(f_{\text {center }}\right)}$ & Strehl ratio & $\eta_{\text {abs }}\left(f_{\text {center }}\right)$ & Polarization & $\frac{\Delta f}{f_{\text {center }}}$ & N.A. \\
\hline Reference $15^{\mathrm{a}}$ & $\sim 1.35 \mu \mathrm{m} / 1.35 \mu \mathrm{m}$ & $\sim 90 \%$ & $\sim 20 \%$ & Circular & $190 \mathrm{THz} / 525 \mathrm{THz}$ & 0.2 \\
\hline Reference $16^{\mathrm{a}}$ & $\sim 2.75 \mu \mathrm{m} / 2.5 \mu \mathrm{m}$ & b & $\sim 50 \%$ & Circular & $195 \mathrm{THz} / 565 \mathrm{THz}$ & 0.106 \\
\hline Reference $30^{\mathrm{a}}$ & $\sim 1.5 \mu \mathrm{m} / 1.46 \mu \mathrm{m}$ & $\sim 80 \%$ & $34 \%$ & Insensitive & $225 \mathrm{THz} / 515 \mathrm{THz}$ & 0.2 \\
\hline Reference $34^{\mathrm{a}}$ & $\sim 800 \mathrm{~nm} / 760 \mathrm{~nm}$ & N.A. too high & b & Insensitive & $35 \mathrm{THz} / 230 \mathrm{THz}$ & 0.88 \\
\hline Reference $35^{\mathrm{a}}$ & $\sim 4.28 \mu \mathrm{m} / 4.28 \mu \mathrm{m}$ & $\sim 85 \%$ & $65 \%$ & Insensitive & $218 \mathrm{THz} / 325 \mathrm{THz}$ & 0.12 \\
\hline Cylindrical MAM lens ${ }^{\mathrm{c}}$ & $1.87 \mu \mathrm{m} / 1.78 \mu \mathrm{m}$ & $73 \%$ & $86 \%$ & Linear & $320 \mathrm{THz} / 545 \mathrm{THz}$ & 0.154 \\
\hline Circular MAM lens ${ }^{c}$ & $1.06 \mu \mathrm{m} / 1.03 \mu \mathrm{m}$ & $96 \%$ & $62 \%$ & Insensitive & $320 \mathrm{THz} / 545 \mathrm{THz}$ & 0.26 \\
\hline
\end{tabular}

${ }^{\mathrm{a}}$ Measurement.

${ }^{\mathrm{b}}$ Not available.

${ }^{\mathrm{c}}$ Simulation.

of which rely on uncoupled metacells. Here, based on our MAM concept, we were able to show polarization-insensitive and efficient operation and achieve fully achromatic focusing in the visible range. While the fabrication process for the designs is relatively demanding, this approach can be conveniently applied, for example, to the infrared region, where the length scales in question are easily reachable with electron-beam lithography. The same challenges may be resolved by utilizing a dielectric of higher refractive index as the pillar material and relaxing the high aspect ratio requirements as the same phase is acquired in a smaller distance. The findings here altogether indicate that continuously tuning the coupling of identical nanopillars in a two-dimensional lattice provides a highly effective approach to make efficient metasurfaces.

See the supplementary material for simulation and implementation details of MAM lenses, as well as the definitions of figuresof-merit used in the text.

The authors gratefully acknowledge the financial support in part from the Singapore National Research Foundation under the programs of Nos. NRF-NRFI2016-08 and NRF-CRP14-2014-03 and the Science and Engineering Research Council, Agency for Science, Technology, and Research $(\mathrm{A} * \mathrm{STAR})$ of Singapore and in part from Nos. TUBITAK 115F297, 117E713, and 119N343. H.V.D. gratefully acknowledges support from TÜBA. Also, the authors thank Mr. İbrahim Tanriöver for his assistance in the early phase of this work, in which he developed another implementation for the concept and ideas of this work proposed by H.V.D. H.B.Y. also thanks Mr. Alper Ahmetoğlu and Mr. Tevfik Bülent Kanmaz for fruitful discussions in stacking optimization.

\section{DATA AVAILABILITY}

The data that support the findings of this study are available from the corresponding author upon reasonable request.

\section{REFERENCES}

${ }^{1}$ F. Bayatpur and K. Sarabandi, "A tunable metamaterial frequency-selective surface with variable modes of operation," IEEE Trans. Microwave Theory Tech. 57, 1433-1438 (2009).

${ }^{2}$ X. Ding, F. Monticone, K. Zhang, L. Zhang, D. Gao, S. N. Burokur, A. de Lustrac, Q. Wu, C.-W. Qiu, and A. Alù, "Ultrathin Pancharatnam-Berry metasurface with maximal cross-polarization efficiency," Adv. Mater. 27, 1195-1200 (2015).

${ }^{3}$ A. Pors, M. G. Nielsen, R. L. Eriksen, and S. I. Bozhevolnyi, "Broadband focusing flat mirrors based on plasmonic gradient metasurfaces," Nano Lett. 13, 829-834 (2013).

${ }^{4}$ X. Ni, S. Ishii, A. V. Kildishev, and V. M. Shalaev, "Ultra-thin, planar, Babinetinverted plasmonic metalenses," Light 2, e72 (2013).

${ }^{5}$ F. Aieta, P. Genevet, M. A. Kats, N. Yu, R. Blanchard, Z. Gaburro, and F. Capasso, "Aberration-free ultrathin flat lenses and axicons at telecom wavelengths based on plasmonic metasurfaces," Nano Lett. 12, 4932-4936 (2012).

${ }^{6}$ M. Khorasaninejad, F. Aieta, P. Kanhaiya, M. A. Kats, P. Genevet, D. Rousso, and F. Capasso, "Achromatic metasurface lens at telecommunication wavelengths,” Nano Lett. 15, 5358 (2015).

${ }^{7}$ M. Khorasaninejad, W. T. Chen, R. C. Devlin, J. Oh, A. Y. Zhu, and F. Capasso, "Metalenses at visible wavelengths: Diffraction-limited focusing and subwavelength resolution imaging," Science 352, 1190-1194 (2016).

${ }^{8}$ M. Khorasaninejad, A. Ambrosio, P. Kanhaiya, and F. Capasso, "Broadband and chiral binary dielectric meta-holograms,” Sci. Adv. 2, e1501258 (2016).

${ }^{9}$ X. Ni, A. V. Kildishev, and V. M. Shalaev, "Metasurface holograms for visible light," Nat. Commun. 4, 2807 (2013).

${ }^{10}$ Y. Hu, X. Luo, Y. Chen, Q. Liu, X. Li, Y. Wang, N. Liu, and H. Duan, “3D-integrated metasurfaces for full-colour holography,” Light Sci. Appl. 8, 86 (2019).

${ }^{11}$ F. Yue, D. Wen, J. Xin, B. D. Gerardot, J. Li, and X. Chen, "Vector vortex beam generation with a single plasmonic metasurface," ACS Photonics 3, 1558-1563 (2016).

${ }^{12}$ R. C. Devlin, A. Ambrosio, N. A. Rubin, J. P. B. Mueller, and F. Capasso, "Arbitrary spin-to-orbital angular momentum conversion of light," Science 358, 896 (2017).

${ }^{13}$ Z. Wang, Y. Yan, A. Arbabi, G. Xie, C. Liu, Z. Zhao, Y. Ren, L. Li, N. Ahmed, A. J. Willner, E. Arbabi, A. Faraon, R. Bock, S. Ashrafi, M. Tur, and A. E. Willner, "Orbital angular momentum beams generated by passive dielectric phase masks and their performance in a communication link," Opt. Lett. 42, 2746-2749 (2017).

${ }^{14}$ A. Arbabi, R. M. Briggs, Y. Horie, M. Bagheri, and A. Faraon, "Efficient dielectric metasurface collimating lenses for mid-infrared quantum cascade lasers," Opt. Express 23, 33310-33317 (2015).

${ }^{15}$ W. T. Chen, A. Y. Zhu, V. Sanjeev, M. Khorasaninejad, Z. Shi, E. Lee, and F. Capasso, "A broadband achromatic metalens for focusing and imaging in the visible," Nat. Nanotechnol. 13, 220 (2018).

${ }^{16}$ S. Wang, P. C. Wu, V. C. Su, Y. C. Lai, M. K. Chen, H. Y. Kuo, B. H. Chen, Y. H. Chen, T. T. Huang, J. H. Wang, R. M. Lin, C. H. Kuan, T. Li, Z. Wang, S. Zhu, and D. P. Tsai, "A broadband achromatic metalens in the visible," Nat. Nanotechnol. 13, 227 (2018).

${ }^{17}$ A. Arbabi, E. Arbabi, Y. Horie, S. M. Kamali, and A. Faraon, "Planar metasurface retroreflector," Nat. Photonics 11, 415 (2017).

${ }^{18}$ A. Cordaro, H. Kwon, D. Sounas, A. F. Koenderink, A. Alù, and A. Polman, "High-index dielectric metasurfaces performing mathematical operations," Nano Lett. 19, 8418-8423 (2019). 
${ }^{19}$ A. Y. Zhu, W. T. Chen, J. Sisler, K. M. Yousef, E. Lee, Y. W. Huang, C. W. Qiu, and F. Capasso, "Compact aberration-corrected spectrometers in the visible using dispersion-tailored metasurfaces," Adv. Opt. Mater. 7, 1801144 (2019).

${ }^{20} \mathrm{M}$. V. Berry, "Quantal phase factors accompanying adiabatic changes," Proc. R. Soc. Lond. A 392, 45-57 (1984).

${ }^{21}$ O. Akin and H. V. Demir, "High-efficiency low-crosstalk dielectric metasurfaces of mid-wave infrared focal plane arrays," Appl. Phys. Lett. 110, 143106 (2017).

${ }^{22}$ R. Paniagua-Domínguez, Y. F. Yu, E. Khaidarov, S. Choi, V. Leong, R. M. Bakker, X. Liang, Y. H. Fu, V. Valuckas, L. A. Krivitsky, and A. I. Kuznetsov, "A metalens with a near-unity numerical aperture," Nano Lett. 18, 2124-2132 (2018).

${ }^{23}$ H. Cai, S. Srinivasan, D. A. Czaplewski, A. B. Martinson, D. J. Gosztola, L. Stan, T. Loeffler, S. K. Sankaranarayanan, and D. López, "Inverse design of metasurfaces with non-local interactions," npj Comput. Mater. 6, 116 (2020).

${ }^{24}$ E. Arbabi, A. Arbabi, S. M. Kamali, Y. Horie, and A. Faraon, "Multiwavelength metasurfaces through spatial multiplexing," Sci. Rep. 6, 1 (2016).

${ }^{25}$ F. Aieta, M. A. Kats, P. Genevet, and F. Capasso, "Multiwavelength achromatic metasurfaces by dispersive phase compensation," Science 347, 1342-1345 (2015).

${ }^{26}$ Z. Shi, M. Khorasaninejad, Y.-W. Huang, C. Roques-Carmes, A. Y. Zhu, W. T. Chen, V. Sanjeev, Z.-W. Ding, M. Tamagnone, K. Chaudhary, R. C. Devlin, C.-W. Qiu, and F. Capasso, "Single-layer metasurface with controllable multiwavelength functions,” Nano Lett. 18, 2420-2427 (2018).

${ }^{27}$ L. Zhang, Q. Lin, Y. Yue, Y. Yan, R. G. Beausoleil, and A. E. Willner, "Silicon waveguide with four zero-dispersion wavelengths and its application in on-chip octave-spanning supercontinuum generation,” Opt. Express 20, 1685-1690 (2012).

${ }^{28}$ G. Y. Lee, J. Y. Hong, S. H. Hwang, S. Moon, H. Kang, S. Jeon, H. Kim, J. H. Jeong, and B. Lee, "Metasurface eyepiece for augmented reality," Nat. Commun. 9, 4562 (2018).

${ }^{29}$ J. P. Balthasar Mueller, N. A. Rubin, R. C. Devlin, B. Groever, and F. Capasso, "Metasurface polarization optics: Independent phase control of arbitrary orthogonal states of polarization," Phys. Rev. Lett. 118, 113901 (2017).

${ }^{30}$ W. T. Chen, A. Y. Zhu, J. Sisler, Z. Bharwani, and F. Capasso, "A broadband achromatic polarization-insensitive metalens consisting of anisotropic nanostructures," Nat. Commun. 10, 355 (2019).

${ }^{31}$ I. Tanriover and H. V. Demir, "Broad-band polarization-insensitive all-dielectric metalens enabled by intentional off-resonance waveguiding at mid-wave infrared," Appl. Phys. Lett. 114, 043105 (2019).

${ }^{32}$ A. W. Snyder and J. D. Love, Optical Waveguide Theory, 1st ed. (Chapman and Hall, 1983), p. 230.

${ }^{33} \mathrm{X}$. Zhao, M. H. Alizadeh, and B. M. Reinhard, "Generating optical birefringence and chirality in silicon nanowire dimers," ACS Photonics 4, 2265-2273 (2017).

${ }^{34}$ S. Shrestha, A. C. Overvig, M. Lu, A. Stein, and N. Yu, "Broadband achromatic dielectric metalenses," Light Sci. Appl. 7, 85 (2018).

${ }^{35}$ A. Ndao, L. Hsu, J. Ha, J. H. Park, C. Chang-Hasnain, and B. Kanté, "Octave bandwidth photonic fishnet-achromatic-metalens," Nat. Commun. 11, 3205 (2020). 